\title{
Enhanced Derivation of the Electron Magnetic Moment Anomaly from the Electron Charge using Geometric Principles
}

\author{
Andrew Worsley ${ }^{1} \&$ J.F. Peters ${ }^{2}$ \\ ${ }^{1}$ UCL, Gower Street, London WC1E 6BT, UK \\ ${ }^{2}$ University of Manitoba, WPG, MB, R3T 5V6, Canada and Adiyaman University, 02040 Adiyaman, Turkey \\ Correspondence: Andrew Worsley, UCL, Gower Street, London WC1E 6BT, UK. E-mail: \\ andrew.worsley@uclmail.net
}

Received: October 24, 2018

Accepted: November 13, 2018

Online Published: November 30, 2018

doi:10.5539/apr.v10n6p24

URL: https://doi.org/10.5539/apr.v10n6p24

\begin{abstract}
The electron magnetic moment anomaly is conventionally derived from the fine structure constant using a complex formula requiring over 13,000 evaluations. However, the charge of the electron is an important parameter of the Standard Model and could provide an enhanced basis for the derivation of the electron magnetic moment anomaly. This paper uses a geometric model to reformulate the equation for the electron's charge, this is then used to determine a more accurate value for the electron magnetic moment anomaly from first geometric principles. This enhanced derivation uses a single evaluation, using a concise mathematical equation based on the natural $\log e^{\pi}$. This geometric model will lead to further work to theoretically improve the understanding of the electron.
\end{abstract}

Keywords: electron magnetic moment anomaly; electron charge; fine structure constant; geometry, natural log

\section{Introduction}

In the Standard model (SM) the electron $g$ factor is related to the electron magnetic moment to Bohr magneton ratio and the electron magnetic moment anomaly, by the following expression:

$$
g / 2=-\mu_{e} / \mu_{B}=1+a_{e},
$$

where $g$ is the electron $g$ factor, $\mu_{e}$ is the electron magnetic moment, $\mu_{B}$ is the Bohr magneton and $a_{\mathrm{e}}$ is the electron magnetic moment anomaly.

The $g$ factor represents a small deviation in quantum mechanics from the equation for the Bohr magneton. For the $g$ factor of the electron, the derivation in the Standard model (Hanneke, Fogwell, \& Gabrielse, 2008), is given by:

$$
g=2\left[1+C_{2}\left(\frac{\alpha}{\pi}\right)+C_{4}\left(\frac{\alpha}{\pi}\right)^{2}+C_{6}\left(\frac{\alpha}{\pi}\right)^{3}+C_{8}\left(\frac{\alpha}{\pi}\right)^{4}+C_{10}\left(\frac{\alpha}{\pi}\right)^{5} \ldots \ldots \ldots\right] \mathrm{a}_{\mu \tau}+\mathrm{a}_{\text {hadronic }}+\mathrm{a}_{\text {weak }},
$$

The principal term $\alpha$ is the fine structure constant, and the first variable component $\mathrm{C}_{2}=1 / 2$. The conventional value for $C_{4}$ and $C_{6}$ for the electron, are computed analytically using the Riemann zeta function $\zeta_{(x)}$ (Laporta \& Remiddi, 1996).

The value for $\mathrm{C}_{8}$ is derived from a numerical integration technique, with 891 evaluations whose value has recently been revised to -1.9106 (20). Greater accuracy is achieved in the standard model with the further addition of $\mathrm{C}_{10}$, using a similar numerical integration technique requiring a further 12,672 evaluations whose approximate value is 9.16 (58) (Aoyama, Hayakawa, Kinoshita, \& Nio, 2012).

Further adjusting the results using a number of $4^{\text {th }}, 6^{\text {th }}, 8^{\text {th }}$ and $10^{\text {th }}$ order mass-dependent terms, and the additional coupling factors, $a_{\mu \tau}+a_{\text {hadronic }}+a_{\text {weak }}$, brings the theoretical result, given to 13 d.p., as 1.0011596521801 (Quint \& Vogel, 2014).

In this paper a more accurate value for the electron magnetic moment anomaly is derived, with a value to 13 d.p. of 1.0011596521807. This is in agreement with the most recent experimental value of 1.00115965218073 (28) (Quint \& Vogel, 2014). This value is derived on an enhanced basis using an equation based on the charge of the 
electron. A spherical model of the electron charge has previously been derived from the conventional equation for the charge of the electron (Worsley, 2011). Here, we use this equation to derive a single concise term for the electron magnetic moment anomaly, from first geometric principles using the natural $\log e^{\pi}$.

\section{Methods}

All mathematical calculations follow strict standard algebraic and standard mathematical rules. The principal physics proofs are based upon standard physical formulae. The value used for Planck's constant $h=$ $6.6260700408 \times 10^{-34}$, is in accordance with the 2014 CODATA evaluation $6.626070040(81) \times 10^{-34} \mathrm{~m}^{2} \mathrm{~kg} / \mathrm{sec}$, The value used for $\alpha=0.0072973525664$ (17), is also taken from the 2014 CODATA evaluation. Derived values are given to 13 decimal places; intermediate values are given to 15 decimal places.

\subsection{Results: Reformulating the Electron Charge}

In the standard QED the charge of the electron is given as:

$$
e^{2}=\varepsilon_{0} .2 h c \alpha
$$

where $e$ is electron charge, $\alpha$ is the fine structure constant, $h$ is Planck's constant, $c$ is the speed of light, and $\varepsilon_{0}$ is the electric constant (permittivity of free space).

The spherical symmetry of the electron charge is well recognized (Baldomir \& Hammond, 2002; Haddad et al., 2016). In a reformulation of the standard equation, the charge of the electron can be expressed as an equation in the form of a sphere (Worsley, 2011). The fractional quantum Hall effect indicates that the charge of the electron exists in multiples of 1/3e (Seiden, 2005; De-Picciotto et al., 1998; von Klitzing, Dorda, \& Pepper, 1980; Tsui, Stormer, \& Gossard, 1982). So, we can do this for three orthogonal dimensions $\mathrm{x}, \mathrm{y}$ and $\mathrm{z}$ to give three orthogonal spheres. The volume within a sphere is $4 / 3 \pi r^{3}$, and for three spheres the volume would be $3 \times 4 / 3$ $\pi r^{3}$.

Recent experimental design shows that matter in the form of electrons and positrons could be formed purely from E-M photons (Dennis, King, Jack, O'Holleran,, \& Padgett, 2010; Pike, Mackenroth, Hill, \& Rose, 2014). Here, it is shown that a threefold spherical model of the charge of the electron can be derived from the conventional equation for the electron charge, where the charge radius is dictated by the speed of light. Thus we can reformulate the standard equation to give the charge of the electron in terms of the volume of three spheres.

$$
\begin{gathered}
e^{2}=\frac{\varepsilon_{0}}{c^{3}} \cdot 2 h c^{4} \alpha, \\
e^{2}=\frac{\varepsilon_{0}}{4 \pi c^{3}} \cdot 8 \pi h c^{4} \alpha, \\
e^{2}=\frac{\varepsilon_{0}}{3\left(\frac{4}{3} \pi c^{3}\right)^{3}} \cdot 8 \pi h c^{4} \alpha,
\end{gathered}
$$

This equation can be simplified to the term

$$
\begin{gathered}
e^{2}=\frac{\varepsilon_{0}}{3\left(4 / 3 \pi c^{3}\right) \cdot x}, \\
e=\sqrt{\frac{\varepsilon_{0}}{3\left(4 / 3 \pi c^{3}\right) \cdot x}}
\end{gathered}
$$

As we have used the conventional equation for the electron charge this agrees with the dimensions of the charge of the electron. So in Eq. (8), $e$ has the dimensions of coulombs [C].

One important aspect of this equation is that the standard equation for the charge of the electron can be reformulated into two terms. The principal term derives from the volume of three spheres based on the speed of

light and the electric constant $e=\sqrt{\frac{\varepsilon_{0}}{3\left(\frac{4}{3} \pi c^{3}\right) \cdot x}}$. The second term represents a small spin coefficient, $x=1 /$ 
$8 \pi h c^{4} \alpha=1.018722648454255$ (Intermediate values are given to 15 decimal point accuracy). Interestingly this spin coefficient can then be used to derive an entirely dimensionless term for the electron magnetic moment anomaly to a higher degree of accuracy, with a single equation, from first geometric principles using $e^{\pi}$. (see Section 3.2).

\subsection{Results Deriving the Electron Magnetic Moment Anomaly}

Here, the derivation of the electron magnetic moment anomaly can be enhanced using a reformulation of the conventional equation for the electron charge [Eqs. (4-8)]. This derivation yields a term from the electron spin coefficient $x$, given as $x=1 / 8 \pi h c^{4} \alpha$. From here, using a straight forward mathematical cancellation technique, allows the derivation of an entirely dimensionless term for the electron magnetic moment anomaly.

As the term $x$ results from derivation of the charge of the electron squared, for the charge of a single electron the spin coefficient $\mathrm{y}$, should be derived from the square root of $x$, hence:

$$
y=x^{1 / 2}-1
$$

This gives the numerical value for $\mathrm{y}=0.00931793031444$ to 15 decimal points. It is stressed that although the term $x$ and in turn $y$ have dimensions, it is possible to derive an equation which is dimensionless by using the term $y / y$, as in Eq. (10) below, thereby cancelling the dimensions.

In accordance with particle gauge coupling (Evans, French, Jensen, \& Threlfall, 2010), the ratio in curved space is $\left(\mathrm{r}_{\mathrm{w}} / \mathrm{r}\right)^{8}$, gives the power ratio of the electron magnetic moment anomaly $\left(1+a_{e}\right)^{8}$. Thus a far more straight forward and accurate dimensionless formula for the anomalous electron magnetic moment, can be derived:

For the electron magnetic moment anomaly to 13 d.p. $\left(a_{e}\right)$ :

$$
1+a_{e}=\sqrt[8]{\frac{y}{\left[\frac{y}{e^{\pi \cdot \sqrt[8]{\pi^{\frac{1}{2}}}}}+1\right]}+1}=1.0011596521807,
$$

Conventionally this gives a dimensionless parameter, where the electron magnetic moment anomaly principally depends on the term y derived from conventional formula for the charge of the electron, and the natural $\log e^{\pi}$.

The CODATA experimental value for the electron magnetic moment to Bohr magneton ratio, given to 11 decimal places is: $\mu_{e} / \mu_{B}=-1.00115965218$.

So the electron magnetic moment to Bohr magneton ratio is accurately related directly to a spin coefficient $\mathrm{y}$, and $e^{\pi}$. The majority of the relative standard uncertainty $(0.00000000075)$ relates to the uncertainty in the value of Planck's constant. Thus, far greater accuracy could be achieved for this equation [Eq. (11)], when Planck's constant is known to a greater degree of accuracy.

\section{Discussion and Conclusion}

With direct concepts of geometry, it possible to enhance the derivation of the electron magnetic moment anomaly, from first principles using the term $e^{\pi}$. Also known as Gelfond's constant, $e^{\pi}$ is a transcendental number with profound mathematical significance, where $e^{\pi}$ represents the sum of all unit-ball volumes of even dimensions.

In the standard model the Hamiltonian of an electron is

$$
\hat{H}=\frac{1}{2 m}(\hat{p}-q \hat{A})^{2}-\hat{\mu} \cdot B,
$$

where $\mathrm{m}$ is the mass of an electron, charge $\mathrm{q}$, in a uniform static magnetic field B directed along the $\mathrm{z}$-axis, $\hat{A}=B \times \frac{\hat{r}}{2}$ is the vector potential and $\hat{\mu}$ is the intrinsic magnetic moment operator of the electron. The magnetic moment $\hat{\mu}$ is related to the spin operator $\hat{S}$ by $\hat{\mu}=(1+a) \frac{q}{m} \hat{S}$, where $a$, is called the electron magnetic moment anomaly (Basdevant \& Dalibard, 2006). 
Here, the electron magnetic moment anomaly is derived from first geometric principles using $e^{\pi}$. Firstly, the standard equation for the charge of the electron can be reformulated to form a spherical model of the electron charge. This reformulation of the elementary charge then yields an electron spin coefficient. From here, a single concise equation is used to derive the electron magnetic moment anomaly, to a greater degree of accuracy than current theory allows. As spin depends upon the dimension of time, the electron charge has spin in 4 dimensions, so the results are explained with geometry using $e^{\pi}$, representing the sum of all unit-ball volumes of even dimensions.

In addition to the deep mathematical significance of $e^{\pi}$, it also appears that Planck's constant $h$ has a profound physical significance. Indeed Planck's constant and the speed of light $c$ are intimately interconnected, not just with each other, but with the very nature of the electron and matter itself. Furthermore, it is possible to redefine a minimum quantum mass $m_{q}$ by the equation

$$
m_{q}=\sqrt{\frac{h c}{G}} \times \sqrt{\frac{h G}{c^{5}}}=\frac{h}{c^{2}},
$$

where $G$ the gravitational constant cancels. This is also the equation for the standard Planck mass multiplied by Planck time. This minimum quantum mass $m_{q}$ can also be used to further explain the equations for quantum electrodynamics (Worsley, 2010).

Overall, given that Planck's constant is only accurately known to 9 decimal places, it represents a notable achievement to be able to obtain 13 decimal place accuracy compared to the known experimental values. Far greater accuracy than this could be achieved for these derivations, when Planck's constant is known more accurately. These insights should lead to other work to improve the understanding of the electron.

Acknowledgements: This paper was supported by Instituto Nazionale di Alta Matematica (INdAM) Francesco Severi, Gruppo Nazionale per le Strutture Algebriche, 140 Geometriche e Loro Applicazioni grant 9920160 000362, n.prot U 2016/000036, the Scientific and Technological Research Council of Turkey (TUB" 'ITAK) Scientific Human Resources Development (BIDEB) under grant no: 2221-1059B211402463, and the Natural Sciences \& Engineering Research Council of Canada (NSERC) discovery grant 185986.

\section{References}

Aoyama, T., Hayakawa, M., Kinoshita, T., \& Nio, M. (2012). Tenth-order QED contribution to the electron g- 2 and an improved value of the fine structure constant. Physical Review Letters, 109(11), 111807-111804. https://doi.org/10.1103/PhysRevLett.109.111807

Baldomir, D., \& Hammond, P. (2002). Geometry of Electromagnetic Systems. Oxford: Oxford University Press.

Basdevant, J. L., \& Dalibard, J. (2006). The quantum mechanics solver: how to apply quantum theory to modern physics (2nd ed.). Springer Science \& Business Media.

Dennis, M. R., King, R. P., Jack, B., O’Holleran, K., \& Padgett, M. J. (2010). Isolated optical vortex knots. Nature Physics, 6(2), 118. https://doi.org/10.1038/nphys1504.

De-Picciotto, R., Reznikov, M., Heiblum, M., Umansky, V., Bunin, G., \& Mahalu, D. (1998). Direct observation of a fractional charge. Physica B: Condensed Matter, 249, 395-400. https://doi.org/10.1016/S0921-4526(98)00139-2

Evans, N., French, J., Jensen, K., \& Threlfall, E. (2010). Hadronization at the AdS wall. Physical Review D, 81(6), 066004. https://doi.org/10.1103/PhysRevD.81.066004, arXiv:0908.0407 [hep-th].

Haddad, D., Seifert, F., Chao, L. S., Li, S., Newell, D. B., Pratt, J. R., ... \& Schlamminger, S. (2016). Invited Article: A precise instrument to determine the Planck constant, and the future kilogram. Review of Scientific Instruments, 87(6), 061301. https://doi.org/10.1063/1.4953825

Hanneke, D., Fogwell, S., \& Gabrielse, G. (2008). New measurement of the electron magnetic moment and the fine structure constant. Physical Review Letters, 100(12), 120801. 10.1007/s00454-017-9859-x

Laporta, S., \& Remiddi, E. (1996). The analytical value of the electron (g-2) at order $\alpha 3$ in QED. Physics Letters B, 379(1-4), 283-291. https://doi.org/10.1016/0370-2693(96)00439-X

Pike, O. J., Mackenroth, F., Hill, E. G., \& Rose, S. J. (2014). A photon-photon collider in a vacuum hohlraum. Nature Photonics, 8(6), 434-436. https://doi.org/10.1038/nphoton.2014.95. 180 
Quint, W., \& Vogel, M. (Eds.). (2014). Fundamental physics in particle traps (Vol. 395). Springer-Verlag, Berlin Heidelberg: Springer.

Seiden, A. (2005). Particle Physics: A Comprehensive Introduction. Addison Wesley, San Francisco.

Tsui, D. C., Stormer, H. L., \& Gossard, A. C. (1982). Two-dimensional magnetotransport in the extreme quantum limit. Physical Review Letters, 48(22), 1559. https://doi.org/10.1103/PhysRevLett.48.1559

von Klitzing, K., Dorda, G., \& Pepper, M. (1980). New method for high-accuracy determination of the fine-structure constant based on quantized hall resistance, Phys. Rev. Lett., 45, 494-497. https://doi.org/10.1103/PhysRevLett.45.494

Worsley, A. (2010). The formulation of harmonic quintessence and a fundamental energy equivalence equation. Physics Essays, 23(2), 311-319. https://doi.org/10.4006/1.3392799.

Worsley, A. (2011). Harmonic quintessence and the derivation of the charge and mass of the electron and the proton and quark masses. Physics Essays, 24(2), 240-253. https://doi.org/10.4006/1.3567418. 155

\section{Copyrights}

Copyright for this article is retained by the author(s), with first publication rights granted to the journal.

This is an open-access article distributed under the terms and conditions of the Creative Commons Attribution license (http://creativecommons.org/licenses/by/4.0/). 